\title{
Reactive Power Control Using a Model-Based Predictive Control Strategy Applied to an Indirect Matrix Converter
}

\author{
Diego Rojas, Marco Rivera, Sergio Toledo, Patrick Wheeler,
}

\begin{abstract}
Reactive power is often an issue of concern for the operation of alternating current (AC) electrical power systems. This work aims to apply a model-based predictive control (MPC) to an indirect matrix converter (IMC) to improve the power factor at the input of a converter whilst still having full control of the output current or voltage. Simulation results from a Matlab/Simulink model are presented. The objective is to compare the control performance of the IMC without input reactive power control and with reactive power minimization.

Keywords-Current control, matrix converter, power converter, predictive control, reactive power, voltage control.
\end{abstract}

\section{INTRODUCCIÓN}

$\mathbf{E}$ $\mathrm{N}$ la familia de convertidores $\mathrm{AC} / \mathrm{AC}$ es posible distinguir dos grupos principales, los convertidores con almacenamiento de energía y aquellos que no lo tienen. En el primer grupo se encuentran las topologías de fuente de corriente y tensión, con las cuales es posible obtener una conversión de AC/AC, pero considerando un elemento almacenador de energía. Estas estructuras han sido ampliamente estudiadas y son los convertidores utilizados en la industria actual [1]. En el grupo de circuitos de AC/AC sin un elemento almacenador de energía, se han reportado diferentes topologías en la literatura, que se clasifican en tres grupos principales: el cicloconvertidor en una amplia variedad de potencia, el convertidor matricial directo (DMC) [2] y el convertidor matricial indirecto (IMC), ambos en rango de baja potencia [3].

El convertidor matricial indirecto (IMC) tiene interesantes propiedades, entre ellas, una corriente de forma sinusoidales tanto en la entrada como en la salida del IMC, la potencia puede fluir en ambas direcciones y el factor de potencia en la entrada del convertidor es ajustable, también, presenta menor dimensión y mayor vida útil que su contraparte el convertidor matricial directo [4]. Además, teniendo en cuenta el control aplicado, el IMC tiene la posibilidad de operar bajo un control

Diego Rojas. Estudiante del Doctorado en Sistemas de Ingeniería, Facultad de Ingeniería, Universidad de Talca, Curicó, Chile, (e-mail: diego.rojaso@utalca.cl).

Marco Rivera. Director del Laboratorio de Conversión de Energías y Electrónica de Potencia, Universidad de Talca, Curicó, Chile, (e-mail: marcoriv@utalca.cl).

Sergio Toledo. Estudiante del Doctorado en Sistemas de Ingeniería, Facultad de Ingeniería, Universidad de Talca, Curicó, Chile, (e-mail: stoledo@ing.una.py).

Patrick Wheeler. Power Electronics, Machines and Control Group, The University of Nottingham, Nottingham, UK, (e-mail: Pat.Wheeler@nottingham.ac.uk).

978-0-7381-3333-1/21\$31.00 @2021 IEEE de corriente de conmutación cero en el enlace de corriente continua (DC-link), que es menos complicado de implementar y menos complejo que los controladores que se usan en el convertidor matricial directo, permitiendo de esta forma que el convertidor pueda conmutar de forma segura, sin la necesidad de implementar esquemas de conmutación seguras muy complejas [5].

Gracias a los avances tecnológicos, se utilizan microprocesadores rápidos y potentes para el control y la modulación de los convertidores de potencia. Este es el caso del control predictivo basado en modelo (MPC) [6], que se basa en el modelo dinámico del sistema para poder anticipar el comportamiento futuro de las variables de control en un horizonte de tiempo predefinido y selecciona la acción óptima teniendo en cuenta la minimización de una función de costo [7]. Esta estructura se caracteriza porque sus conceptos son muy intuitivos y fáciles de entender, se puede aplicar a una amplia variedad de sistemas, puede involucrar múltiples sistemas, compensación de tiempo muerto y restricciones no lineales, lo que resulta en un controlador fácil de implementar, posibilidad de modificaciones y extensiones para aplicaciones específicas, entre otras [8], [9], [10].

En este trabajo se consideran las ventajas del control predictivo y el convertidor matricial indirecto, para controlar de manera sencilla e intuitiva las señales de salida del convertidor al tiempo que garantiza un factor de potencia unitario en la entrada del sistema. El enfoque predictivo se basa en el hecho de que solo un número fijo de conmutaciones factibles puede ser generado por un convertidor de potencia estático y el modelo dinámico pueden usarse para determinar los valores que tendrán las señales a ser controladas para cada estado de conmutación. Para validar las estrategias MPC aplicado al IMC, las simulaciones se llevan a cabo utilizando el software Matlab/Simulink.

\section{PRINCIPIOS BÁSICOS DEL IMC}

La configuración de la topología del convertidor IMC incluye dos etapas que son el rectificador y el inversor [11], así como se observa en la Fig. 1. Esta topología presenta una llamativa ventaja en el caso que se emplee un esquema con la que conmuten los interruptores con corriente cero en el DC-link, lo que proporciona una reducción de la pérdidas de conmutación y logra que el convertidor opere de forma segura. El modelo matemático del rectificador está definido por las expresiones (1) y (2). 


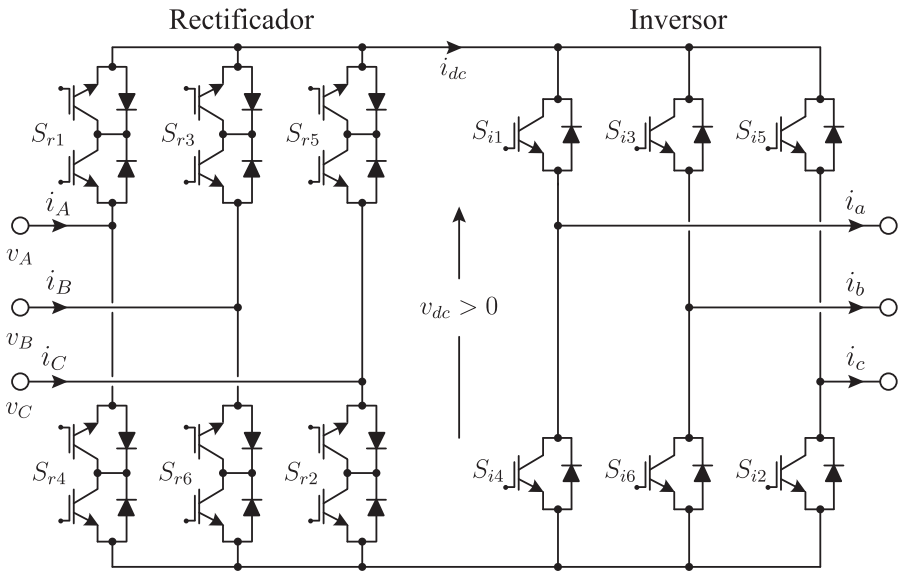

Fig. 1. Topología del convertidor matricial indirecto.

$$
\begin{aligned}
& v_{d c}=\left[\begin{array}{lll}
S_{r 1}-S_{r 4} & S_{r 3}-S_{r 6} & S_{r 5}-S_{r 2}
\end{array}\right] v_{i} \\
& i_{i}=\left[\begin{array}{l}
S_{r 1}-S_{r 4} \\
S_{r 3}-S_{r 6} \\
S_{r 5}-S_{r 2}
\end{array}\right] i_{d c}
\end{aligned}
$$

donde $v_{i}=\left[\begin{array}{l}v_{A} \\ v_{B} \\ v_{C}\end{array}\right], \mathrm{e} i_{i}=\left[\begin{array}{l}i_{A} \\ i_{B} \\ i_{C}\end{array}\right]$ son la tensión de entrada en el rectificador y la corriente de entrada de esta etapa, respectivamente. Para los estados de conmutación, es relevante describir tanto las entradas como salidas de ambas etapas. Para la etapa del inversor, la dinámica está definido por las expresiones (3) y (4):

$$
\begin{aligned}
& i_{d c}=\left[\begin{array}{lll}
S_{i 1} & S_{i 3} & S_{i 5}
\end{array}\right] i_{o} \\
& v_{o}=\left[\begin{array}{l}
S_{i 1}-S_{i 4} \\
S_{i 3}-S_{i 6} \\
S_{i 5}-S_{i 2}
\end{array}\right] v_{d c}
\end{aligned}
$$

donde $v_{o}=\left[\begin{array}{l}v_{a} \\ v_{b} \\ v_{c}\end{array}\right]$, e $i_{o}=\left[\begin{array}{l}i_{a} \\ i_{b} \\ i_{c}\end{array}\right]$ son la tensión de salida que proporciona el inversor y la corriente de salida de esta etapa, respectivamente.

Por otro lado, de los posibles estados de conmutación, solo son válidos aquellos que cumplan con las siguientes restricciones de operación segura: en la entrada del rectificador la fases no deben cortocircuitarse, de esta forma solo nueve estados son válidos. No deben desconectarse instantáneamente las fases en la salida del inversor, entonces solo es posible utilizar ocho estados válidos. También, hay que tener en cuenta que la tensión del DC-link debe ser positiva para lograr que los interruptores del inversor conmuten. De esta forma, el rectificador presenta solo tres estados válidos de conmutación en cada instante de muestreo. Finalmente el IMC presenta 24 estados válidos.

En la Tabla I y la Tabla II se presenta un resumen de cada estado válido para el lado del rectificador y para el inversor del IMC, respectivamente, así como su relación en términos de corrientes y tensiones [12].

Tabla I

ESTADOS VÁLIDOS DEL LADO DEL RECTIFICADOR.

\begin{tabular}{c|cccccc|ccc}
\hline$\#$ & $S_{r 1}$ & $S_{r 2}$ & $S_{r 3}$ & $S_{r 4}$ & $S_{r 5}$ & $S_{r 6}$ & $i_{A}$ & $i_{B}$ & $i_{C}$ \\
\hline 1 & 1 & 1 & 0 & 0 & 0 & 0 & $i_{d c}$ & 0 & $-i_{d c}$ \\
2 & 0 & 1 & 1 & 0 & 0 & 0 & 0 & $i_{d c}$ & $-i_{d c}$ \\
3 & 0 & 0 & 1 & 1 & 0 & 0 & $-i_{d c}$ & $i_{d c}$ & 0 \\
4 & 0 & 0 & 0 & 1 & 1 & 0 & $-i_{d c}$ & 0 & $i_{d c}$ \\
5 & 0 & 0 & 0 & 0 & 1 & 1 & 0 & $-i_{d c}$ & $i_{d c}$ \\
6 & 1 & 0 & 0 & 0 & 0 & 1 & $i_{d c}$ & $-i_{d c}$ & 0 \\
\hline
\end{tabular}

Tabla II

ESTADOS VÁLIDOS DEL LADO DEL INVERSOR.

\begin{tabular}{c|cccccc|ccc}
\hline$\#$ & $S_{i 1}$ & $S_{i 2}$ & $S_{i 3}$ & $S_{i 4}$ & $S_{i 5}$ & $S_{i 6}$ & $v_{u v}$ & $v_{v w}$ & $v_{w u}$ \\
\hline 1 & 1 & 1 & 0 & 0 & 0 & 1 & $v_{d c}$ & 0 & $-v_{d c}$ \\
2 & 1 & 1 & 1 & 0 & 0 & 0 & 0 & $v_{d c}$ & $-v_{d c}$ \\
3 & 0 & 1 & 1 & 1 & 0 & 0 & $-v_{d c}$ & $v_{d c}$ & 0 \\
4 & 0 & 0 & 1 & 1 & 1 & 0 & $-v_{d c}$ & $v_{d c}$ & 0 \\
5 & 0 & 0 & 0 & 1 & 1 & 1 & 0 & $-v_{d c}$ & $v_{d c}$ \\
6 & 1 & 0 & 0 & 0 & 1 & 1 & $v_{d c}$ & $-v_{d c}$ & 0 \\
7 & 1 & 0 & 1 & 0 & 1 & 0 & 0 & 0 & 0 \\
8 & 0 & 1 & 0 & 1 & 0 & 1 & 0 & 0 & 0 \\
\hline
\end{tabular}

\section{Control MPC Aplicado Al IMC}

La técnica MPC, consiste en determinar el comportamiento futuro de las variables de interés en el sistema analizado, para ello se hace uso del modelo que describe la dinámica del sistema, se evalúa para cada estado de conmutación valido del convertidor y se elige aquel que de mejor manera cumpla con los objetivos deseados [13]. De este modo, el primer paso para implementar el control consiste en modelar el sistema de interés. El modelo del sistema propuesto para la implementación en simulación se muestra en la Fig. 2.

\section{A. Modelo del filtro de entrada}

El rectificador incluye un filtro LC en la entrada que es necesario para evitar sobretensiones y para proporcionar filtrado de las componentes de alta frecuencia de las corrientes de entrada producidas por las conmutaciones [14].

Por la ley de tensión de Kirchhoff y aproximaciones de la derivada [15], [16], se obtiene la siguiente expresión para la estimación de la corriente futura $i_{s}(k+1)$ :

$$
i_{s}(k+1)=\left(1-\frac{R_{f} T_{s}}{L_{f}}\right) i_{s}(k)-\frac{T_{s}}{L_{f}}\left(v_{i}(k)-v_{s}(k)\right)
$$

\section{B. Modelo del filtro de salida}

Luego, en el lado de la carga, la expresión que estima la corriente futura es:

$$
i_{o}(k+1)=\left(1-\frac{R T_{s}}{L}\right) i_{o}(k)-\frac{T_{s}}{L}\left(v_{c}(k)-v_{o}(k)\right)
$$

La estimación de la tensión futura se define como:

$$
v_{c}(k+1)=v_{c}(k)+\frac{T_{s}}{C} i_{o}(k+1)-\frac{T_{s}}{C} i_{g}(k)
$$




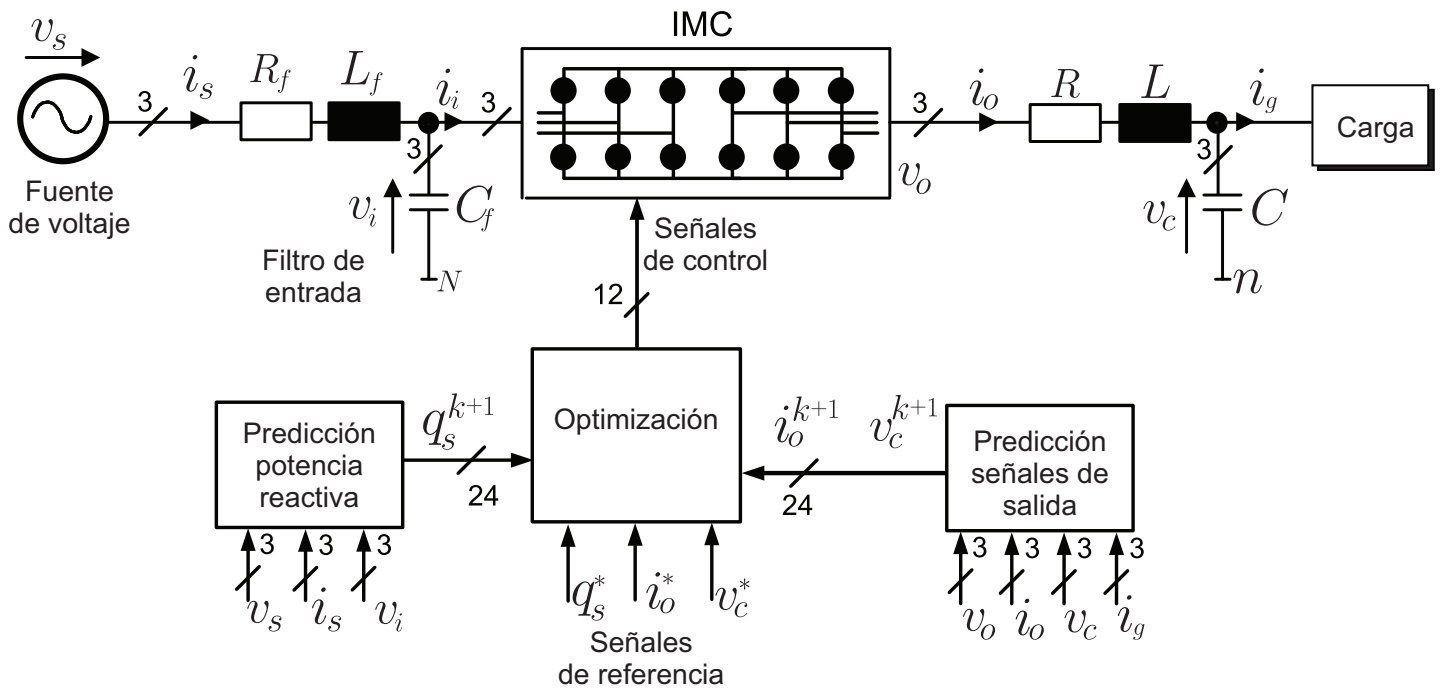

Fig. 2. Esquema propuesto para la implementación del control MPC aplicado al IMC.

Siendo:

$$
i_{o}=\left[\begin{array}{l}
i_{a} \\
i_{b} \\
i_{c}
\end{array}\right] v_{o}=\left[\begin{array}{l}
v_{a} \\
v_{b} \\
v_{c}
\end{array}\right] v_{c}=\left[\begin{array}{l}
v_{c a} \\
v_{c b} \\
v_{c c}
\end{array}\right]
$$

\section{La potencia reactiva instantánea}

El desempeño del convertidor se puede mejorar considerando la potencia reactiva instantánea de entrada $Q_{i n}$ en la red trifásica [17]. Esta potencia reactiva se calcula mediante la siguiente ecuación:

$$
Q_{\text {in }}=\operatorname{Im}\left\{v_{s}(t) \cdot \bar{i}_{s}(t)\right\}
$$

Im corresponde a la parte imaginaria del producto de los vectores $v_{s}(t) \cdot \bar{i}_{s}(t)$ e $\bar{i}_{s}(t)$ es el complejo conjugado de $i_{s}(t)$. La potencia reactiva instantánea de entrada se puede predecir en el plano $(\alpha-\beta)$ mediante el uso de la ecuación (9) [18]:

$$
Q_{\text {in }}(k+1)=v_{s \beta}(k+1) \cdot i_{s \alpha}(k+1)-v_{s \alpha}(k+1) \cdot i_{s \beta}(k+1)
$$

El valor de la corriente de entrada predicha $i_{s}(k+1)$ se obtiene a partir de la ecuación (5). Las tensiones de línea son señales de baja frecuencia y se puede considerar que $v_{s}(k+1) \approx v_{s}(k)$.

\section{Función de costo}

1) Control predictivo de corriente: de manera a definir una función de costo que tiene como objetivo el seguimiento de corriente, se define la corriente de referencia $i_{o}^{*}$ y se compara con las corrientes predichas $i_{o}^{p}$ por la ecuación (6). La función de costo planteada para lograr el control de corriente y reducir la potencia reactiva es [19]:

$$
g=\left(i_{o}^{*}-i_{o}^{p}\right)^{2}+A \cdot\left|Q_{i n}^{*}-Q_{i n}^{p}\right|
$$

donde $Q_{i n}^{p}$ es la potencia reactiva de entrada predicha que se obtiene para cada estado de conmutación válido y se calcula por la ecuación (9), $Q_{i n}^{*}$ es el valor de referencia de la potencia reactiva de entrada, en la mayoría de las aplicaciones se requiere un factor de potencia unitaria por lo tanto $Q_{i n}^{*}=0$. " $A$ " es el factor de ponderación que maneja la relevancia del control de potencia reactiva [20].

2) Control predictivo de tensión: para definir la función de costo que tiene como objetivo el seguimiento de tensión, se define la tensión de referencia $v_{c}^{*}$ y se compara con las tensiones predichas $v_{c}^{p}$ por la ecuación (7). La función de costo es la siguiente [21], [22]:

$$
g=\left(v_{c}^{*}-v_{c}^{p}\right)^{2}+A \cdot\left|Q_{i n}^{*}-Q_{i n}^{p}\right|
$$

siendo $v_{c}^{*}$ la tensión de referencia (la deseada) en el capacitor del filtro de salida, y $v_{c}^{p}$ la tensión predicha por el algoritmo de control sobre el capacitor del filtro de salida, donde $Q_{i n}^{p}$ es la potencia reactiva de entrada predicha que se obtiene para cada estado de conmutación válido y se calcula por la ecuación (9), se tiene que $Q_{i n}^{*}=0$ de tal forma a considerar un caso igual al anterior para obtener un factor de potencia unitario. La ponderación de la reducción de la potencia reactiva es controlada por el parámetro $A$.

\section{Resultados de Simulación}

La técnica de control predictivo sin y con minimización de potencia reactiva son simuladas para evaluar el efecto de introducir la minimización de potencia reactiva en el esquema de control. En primera instancia se realiza el control de corriente, luego, el control de tensión. La simulación del control predictivo expuesto sobre el convertidor matricial indirecto se realizó en el entorno de simulación Matlab/Simulink.

Los parámetros de control se indican en la Tabla III y el período de muestreo del algoritmo de control se estableció en $T_{s}=10[\mu s]$.

\section{A. Control de corriente}

1) Resultados de simulación sin minimización de potencia reactiva: primero, el esquema de control se simula sin incluir el término que minimiza la potencia reactiva en el sistema 
Tabla III

PARÁMETROS DE SIMULACIÓN.

\begin{tabular}{c|c|c|c}
\hline Variable & Descripción & Valor & Unidad \\
\hline$v_{s}$ & Tensión de la fuente & 311 & Vrms \\
$f_{s}$ & Frecuencia de la fuente & 50 & $H z$ \\
$T_{s}$ & Tiempo de muestreo & 10 & $\mu s$ \\
$R_{g}$ & Resistencia de carga & 17 & $\Omega$ \\
$L$ & Induct. del filtro de salida & 10 & $m H$ \\
$R$ & Resist. del filtro de salida & 0.5 & $\Omega$ \\
$C$ & Capacit. del filtro de salida & 25 & $\mu F$ \\
$L_{f}$ & Induct. del filtro de entrada & 400 & $\mu H$ \\
$C_{f}$ & Capacit. del filtro de entrada & 21 & $\mu F$ \\
$R_{f}$ & Resist. del filtro de entrada & 0.5 & $\Omega$ \\
$R_{d}$ & Resist. de amortiguamiento & 50 & $\Omega$ \\
$i^{*}$ & Corriente de referencia & 6 & $A$ \\
$v^{*}$ & Tensión de referencia & 220 & $V$ \\
$f_{r e f}$ & Frecuencia de referencia & 50 & $H z$ \\
$Q_{i n}^{*}$ & Ref. de pot. reactiva & 0 & $V A$ \\
$A_{c}$ & Factor de peso de corriente & 0.033 & \\
$A_{v}$ & Factor de peso de tensión & 0.1 & \\
\hline
\end{tabular}

a)

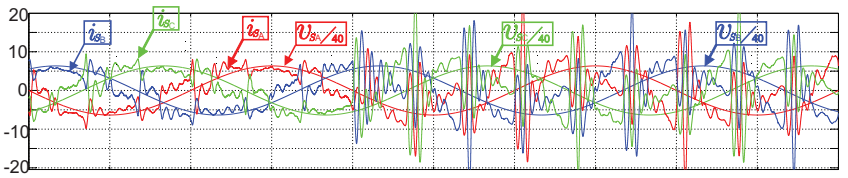

b)

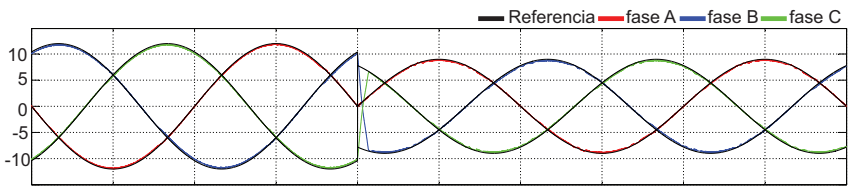

c)

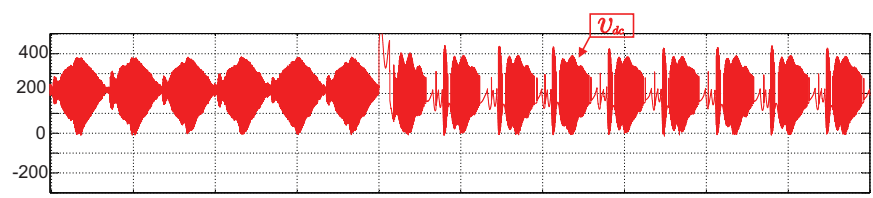

d)

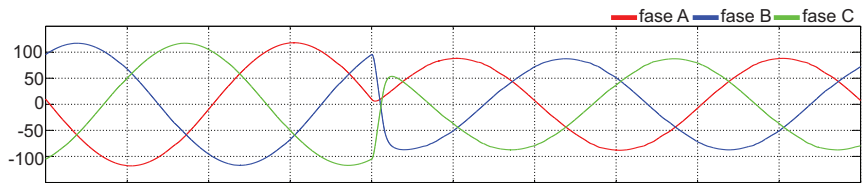

e)

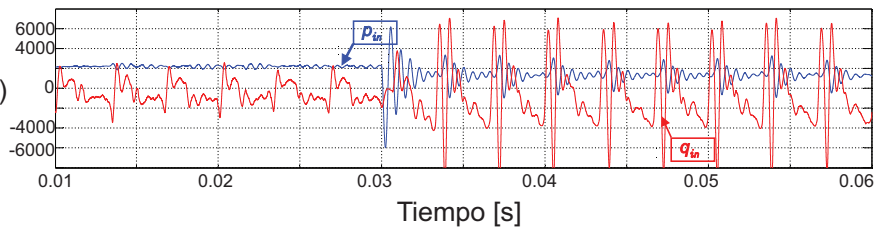

Fig. 3. Resultados de la simulación del control de corriente sin minimización de potencia reactiva en estado transitorio, a) $i_{s}$ : corriente de la fuente y $v_{s} / 40$ : tensión de la fuente, $b$ ) corriente de salida, $c$ ) DC-link (V), d) tensión sobre la carga, e) $Q_{i n}$ : potencia reactiva de entrada.

de entrada. El resultado muestra la distorsión en la corriente de entrada y en desfasaje con la tensión de la fuente. En la Fig. 3 se muestra el comportamiento ante un cambio en la magnitud de la referencia a los $t=0.03[s]$. Observando un buen seguimiento de las corrientes de salida a su respectivas referencias, sin un efecto transitorio considerable cuando se aplica un cambio de referencia Fig. 3.b. a)

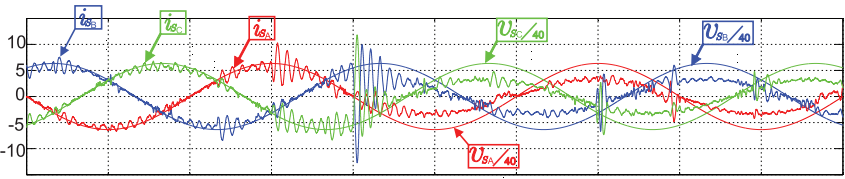

b)

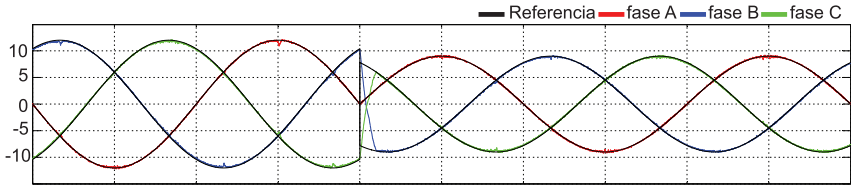

c)

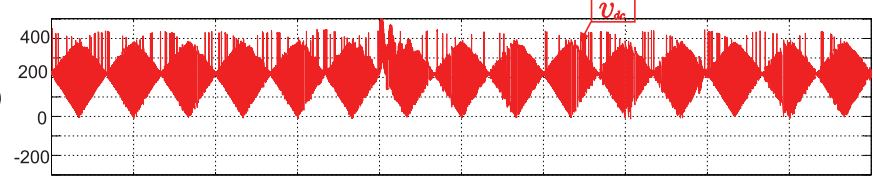

d)
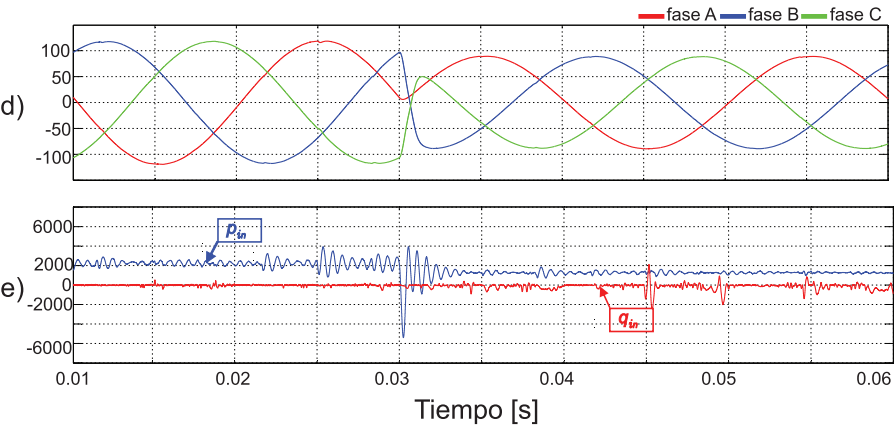

Fig. 4. Resultados de la simulación del control de corriente con minimización de potencia reactiva en estado transitorio, $a) i_{s}$ : corriente de la fuente y $v_{s}$ /40: tensión de la fuente, $b$ ) corriente de salida, $c$ ) DC-link (V), $d$ ) tensión sobre la carga, e) $Q_{i n}$ : potencia reactiva de entrada.

2) Resultados de simulación con minimización de potencia reactiva: en el segundo caso, la estrategia de control se evalúa considerando dos términos en la función de costo, la minimización de la potencia reactiva en el lado de entrada y el control de corriente de salida. Los resultados de la simulación se representan en la Fig. 4, que representa el resultado en estado transitorio para un cambio en la magnitud de la señal de referencia.

Se aprecia un excelente comportamiento de la entrada, con la corriente casi sinusoidal en fase correcta su respectiva tensión de entrada, que es la condición de factor de potencia unitario Fig. 4.a. Además, se observa el buen seguimiento de la corriente de salida a su respectiva referencia Fig. 4.b. En el resultado ante un cambio de la magnitud de referencia se puede observar una ondulación después de $t=0.03[s]$ debido a la resonancia del filtro. El factor $A$ de la función de costo, ecuación (10) se estableció en 0.033 que ha sido ajustado empíricamente. La Fig. 4.e muestra la mejora sobre la minimización de la potencia reactiva de entrada considerando la escala observada en la figura, por lo tanto, el objetivo del control predictivo de corriente se verifica claramente.

\section{B. Control de tensión}

1) Resultados de simulación sin minimización de potencia reactiva: primero, el esquema de control se simula sin incluir el término $(A)$ que minimiza la potencia reactiva en la entrada 
a)

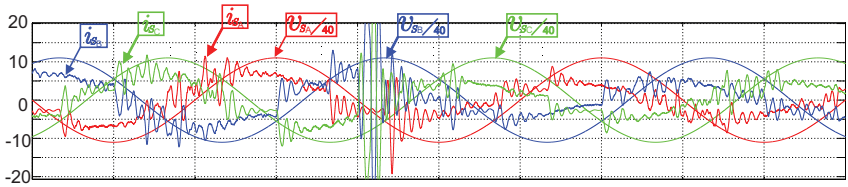

b)
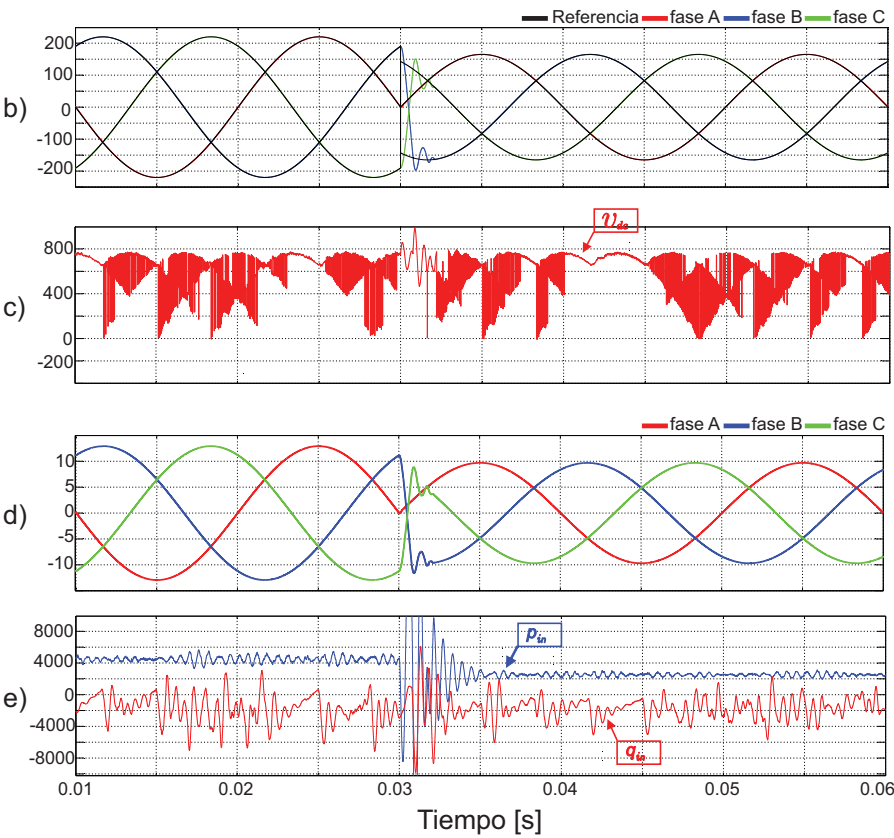

Fig. 5. Resultados de la simulación del control de tensión sin minimización de potencia reactiva en estado transitorio, $a) i_{s}$ : corriente de la fuente y $v_{s} / 40$ : tensión de la fuente, $b$ ) tensión de salida, $c$ ) DC-link (V), $d$ ) corriente sobre la carga, e) $Q_{i n}$ : potencia reactiva de entrada.

del sistema, considerando la función de costo de la ecuación (11). El resultado muestra la distorsión en la corriente de entrada y en desfase con la tensión de la fuente. En la Fig. 5 se observa el comportamiento ante un cambio en la magnitud de la referencia en el tiempo $t=0.03[s]$, respectivamente. En la Fig. 5.b, se observa la señales de las tensiones de salida con sus respectivas referencias, logrando un buen seguimiento sin un efecto transitorio considerado cuando se aplica el cambio de magnitud.

A continuación, se analiza el mismo esquema, pero con el aporte de minimizar la potencia reactiva en la entrada del sistema. Debe recordarse que la principal característica del método de control propuesto es la simplicidad de la implementación, ya que el controlador no necesita una unidad de modulación compleja, lo que puede reducir el costo total del sistema.

2) Resultados de simulación con minimización de potencia reactiva: en el segundo caso, la estrategia de control se evalúa considerando dos términos en la función de costo, la minimización de la potencia reactiva en el lado de entrada y el control de tensión de salida. El resultado de la simulación se representa en la Fig. 6, que representa el resultado en estado transitorio para un cambio en la magnitud de la señal de referencia. Se aprecia un excelente comportamiento de la entrada, con la corriente casi sinusoidal en fase correcta a)
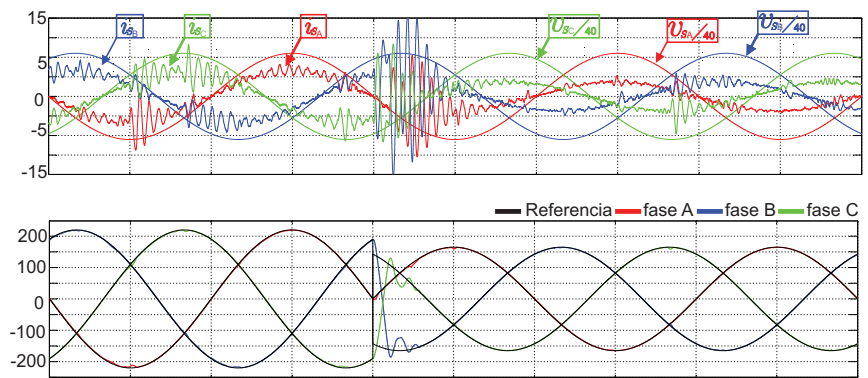

c)

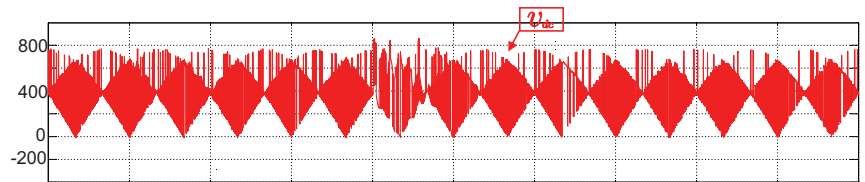

d)
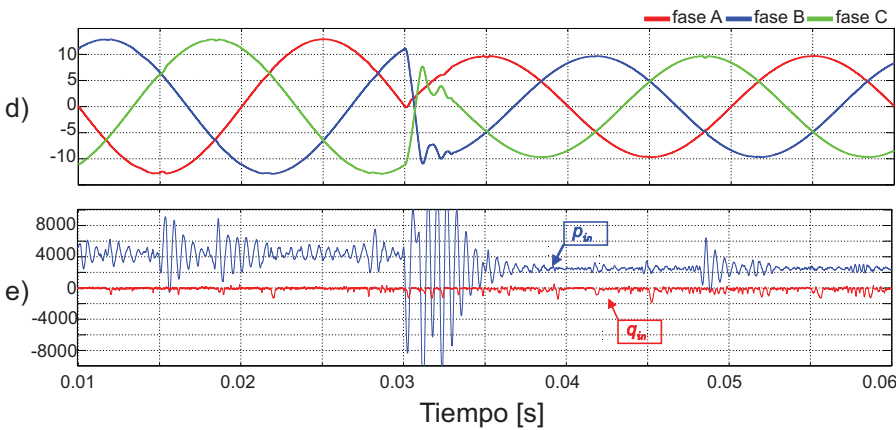

Fig. 6. Resultados de la simulación del control de tensión con minimización de potencia reactiva en estado transitorio, $a) i_{s}$ : corriente de la fuente y $v_{s} / 40$ : tensión de la fuente, $b$ ) tensión de salida, $c$ ) DC-link (V), $d$ ) corriente sobre la carga, e) $Q_{i n}$ : potencia reactiva de entrada.

con la tensión de fase de entrada, que es la condición de factor de potencia unitario Fig. 6.a. Se observa el buen seguimiento de la tensión de salida a sus respectivas referencias Fig. 6.b. En el resultado ante un cambio de la magnitud de referencia se puede observar una ondulación después de $t=0.03[s]$ debido a la resonancia del filtro. El factor $A$ de la función de costo de la ecuación (11) se estableció en 0.1 que ha sido ajustado empíricamente. La Fig. 6.e muestra la mejora sobre la minimización de la potencia reactiva de entrada considerando la escala observada en la figura, por lo tanto, el objetivo del control predictivo de tensión se verifica claramente.

\section{CONCLUSiOnES}

Este trabajo presentó la estrategia de control predictivo basado en modelo para un convertidor matricial indirecto, para aplicaciones tanto de control como de corriente en la salida con minimización de potencia reactiva. La estrategia presentada permite regular la entrada del factor de potencia mediante una técnica simple y directa, controlando la fase de la corriente de entrada de manera a que el convertidor pueda trabajar con potencia capacitiva, unitaria o inductiva, de acuerdo con los requisitos de la aplicación. La técnica de control propuesta presento un buen desempeño desde el punto de vista de la dinámica pues los transitorios obtenidos 
son lo suficientemente cortos, y en estado estacionario pues el seguimiento a la referencia es logrado en dicho estado. El uso del IMC permite implementar dicho algoritmo de control de manera más simple y menos compleja en comparación con el convertidor matricial directo.

\section{AGRADECIMIENTOS}

Los autores agradecen el apoyo económico del Proyecto de Investigación FONDECYT Regular 1191028, MEC 80190074, MEC 801800974 y FONDAP SERC Chile 15110019.

\section{REFERENCIAS}

[1] F. Grimm and M. Baghdadi, "Variable switching point model predictive control for dc-link capacitance minimization of back-to-back converters," in 2020 27th International Workshop on Electric Drives: MPEI Department of Electric Drives 90th Anniversary (IWED), 2020, pp. 16.

[2] S. Feng, C. Wei, and J. Lei, "Reduction of prediction errors for the matrix converter with an improved model predictive control," Energies, vol. 12, no. 15, p. 3029, 2019.

[3] K. Kumari and A. K. Sharma, "Performance analysis of symmetric transformerless buck boost ac-ac converter," in 2019 2nd International Conference on Power Energy, Environment and Intelligent Control (PEEIC), 2019, pp. 342-346.

[4] K. Okuzono, S. Tomita, and H. Haga, "Expand output voltage range of ac/ac converter using reversible indirect matrix converter (r-imc)," in 2019 IEEE Energy Conversion Congress and Exposition (ECCE), 2019, pp. 3587-3592.

[5] J. Jongudomkarn, J. Liu, Y. Yanagisawa, H. Bevrani, and T. Ise, "Model predictive control for indirect boost matrix converter based on virtual synchronous generator," IEEE Access, vol. 8, pp. 60364-60 381, 2020.

[6] S. Borreggine, V. G. Monopoli, G. Rizzello, D. Naso, F. Cupertino, and R. Consoletti, "A review on model predictive control and its applications in power electronics," in 2019 AEIT International Conference of Electrical and Electronic Technologies for Automotive (AEIT AUTOMOTIVE). IEEE, 2019, pp. 1-6.

[7] R. E. Pérez-Guzmán, M. Rivera, and P. W. Wheeler, "Recent advances of predictive control in power converters," in 2020 IEEE International Conference on Industrial Technology (ICIT), 2020, pp. 1100-1105.

[8] S. Toledo, M. Rivera, P. Wheeler, T. Dragicevic, D. Caballero, R. Gregor, E. Maqueda, and F. Gavilán, "Active and reactive power control based on an inner predictive voltage control loop for ac generation systems with direct matrix converter," in 2019 IEEE International Autumn Meeting on Power, Electronics and Computing (ROPEC), 2019, pp. 1-6.

[9] S. Hanke, O. Wallscheid, and J. Böcker, "Continuous-control-set model predictive control with integrated modulator in permanent magnet synchronous motor applications," in 2019 IEEE International Electric Machines Drives Conference (IEMDC), 2019, pp. 2210-2216.

[10] M. Khosravi, M. Amirbande, D. A. Khaburi, M. Rivera, J. Riveros, J. Rodriguez, A. Vahedi, and P. Wheeler, "Review of model predictive control strategies for matrix converters," IET Power Electronics, vol. 12, no. 12 , pp. $3021-3032,2019$
[11] J. Rodriguez, J. Kolar, J. Espinoza, M. Rivera, and C. Rojas, "Predictive current control with reactive power minimization in an indirect matrix converter," in 2010 IEEE International Conference on Industrial Technology, 2010, pp. 1839-1844.

[12] A. Farhadi, A. Zakerian, and A. Nazari, "Predictive control of neutralpoint clamped indirect matrix converter," in 2017 Iranian Conference on Electrical Engineering (ICEE), 2017, pp. 1406-1411.

[13] Z. Malekjamshidi, M. Jafari, J. Zhu, and D. Xiao, "Comparative analysis of input power factor control techniques in matrix converters based on model predictive and space vector control schemes," IEEE Access, vol. 7 , pp. $139150-139160,2019$.

[14] M. Rivera, J. Rodriguez, C. Garcia, R. Pena, and J. Espinoza, "A simple predictive voltage control method with unity displacement power factor for four-leg indirect matrix converters," in 2012 15th International Power Electronics and Motion Control Conference (EPE/PEMC), 2012, pp. DS2c.5-1-DS2c.5-6.

[15] H. M. Basri and K. Lias, "Predictive current control of three level neutral point clamped based indirect matrix converter," in 2019 International UNIMAS STEM 12th Engineering Conference (EnCon), 2019, pp. 1621

[16] H. M. Basri, K. Lias, and S. Mekhilef, "Digital predictive current control fed by three-level indirect matrix converter under unbalanced power supply condition," in 2019 International UNIMAS STEM 12th Engineering Conference (EnCon), 2019, pp. 34-39.

[17] M. Ishaq, M. H. Afzal, and M. Waqar, "Reactive power compensation and switching optimization in matrix converter using model predictive current control approach," in 2020 International Conference on Emerging Trends in Smart Technologies (ICETST), 2020, pp. 1-6.

[18] M. Gokdag and O. Gulbudak, "Model predictive control of ac-dc matrix converter with unity input power factor," in 2018 IEEE 12th International Conference on Compatibility, Power Electronics and Power Engineering (CPE-POWERENG 2018), 2018, pp. 1-5.

[19] C. F. Garcia, M. E. Rivera, J. R. Rodríguez, P. W. Wheeler, and R. S. Peña, "Predictive current control with instantaneous reactive power minimization for a four-leg indirect matrix converter," IEEE Transactions on Industrial Electronics, vol. 64, no. 2, pp. 922-929, 2017.

[20] M. B. Shadmand, S. Jain, and R. S. Balog, "Autotuning technique for the cost function weight factors in model predictive control for power electronic interfaces," IEEE Journal of Emerging and Selected Topics in Power Electronics, vol. 7, no. 2, pp. 1408-1420, 2019.

[21] S. Feng, J. Lei, J. Zhao, W. Chen, and F. Deng, "Improved reference generation of active and reactive power for matrix converter with mode predictive control under input disturbances," IEEE Access, vol. 7, pp. $97001-97012,2019$

[22] C. Garcia, M. Rivera, J. Rodriguez, and P. Wheeler, "Experimental evaluation of predictive voltage control for a four-leg two-stage matrix converter," IET Power Electronics, vol. 12, no. 12, pp. 3077-3084, 2019. 